\title{
Quantum Computers and Decoherence: Exorcising the Demon from the Machine
}

\author{
Daniel A. Lidar and Lian-Ao Wu \\ Chemical Physics Theory Group, Chemistry Department, University of Toronto, \\ 80 St. George St., Toronto, Ontario M5S 3H6, Canada
}

\begin{abstract}
Decoherence is the main obstacle to the realization of quantum computers. Until recently it was thought that quantum error correcting codes are the only complete solution to the decoherence problem. Here we present an alternative that is based on a combination of a decoherence-free subspace encoding and the application of strong and fast pulses: "encoded recoupling and decoupling" (ERD). This alternative has the advantage of lower encoding overhead (as few as two physical qubits per logical qubit suffice), and direct application to a number of promising proposals for the experimental realization of quantum computers.
\end{abstract}

\section{INTRODUCTION}

In the quest to construct large-scale quantum information processors, in particular quantum computers, decoherence is still the main obstacle to realization. Decoherence is the degradation of quantum information due to inevitable interactions with the environment. Early skepticism ${ }^{1,2}$ concerning the viability of quantum computation (QC) in the presence of decoherence was overcome by the discovery of quantum error correcting codes (QECCs) ${ }^{3-9}$ that flourished into a comprehensive theory that incorporates all elements of quantum computation. ${ }^{10}$ A QECC relies on an encoding of quantum information into the state of several quantum bits (qubits), and a closed-loop, active error diagnosis and correction procedure. ${ }^{11}$ In principle, it is possible to correct arbitrary errors using sufficiently large QECCs. ${ }^{8}$ In practice, however, this may require a very large overhead, especially in terms of qubit resources. ${ }^{9}$ This is troubling in light of the substantial difficulties associated with generating and controlling systems with very large numbers of qubits. In addition, the theory of QECCs is rather abstract, in that it presumes that one can execute certain logical operations, but does not refer to the underlying Hamiltonians governing specific physical systems. Hence it is of interest to explore alternatives to QECCs, that are more economical in qubit resources, and that are direcly tailored to specific quantum computer proposals.

Here we review our recent progress in developing such an alternative, "encoded recoupling and decoupling" (ERD),${ }^{12-21}$ and report on some new results. ERD is based on a combination of encoding quantum information into a decoherence-free subspace (DFS) ${ }^{22-25}$ (for a review see Ref. 26), and the application of fast and strong dynamical-decoupling (or "bang-bang", BB) pulses. ${ }^{15,18,19,21,27-45}$ The DFS encoding provides a first layer of protection against decoherence, while the $\mathrm{BB}$ pulses are used to efficiently reduce the remaining decoherence. The encoded "recoupling" part of ERD refers to the application of control operations that enact universal quantum logic, in a manner that is fully compatible with the DFS encoding and the BB pulses, and takes into account the experimentally available control resources, such as the underlying system Hamiltonian. ${ }^{13}$ The utility of ERD as a general method for quantum simulation, universal QC, and decoherence suppression has also been stressed and explored by Viola. ${ }^{37}$ Our work builds in part on earlier efforts to combine universal QC with DFS encoding (without BB pulses). ${ }^{32,35,46,47}$

The structure of the paper is as follows. We begin in Section 2 with a brief formal summary of decoherence. We then give in Section 3 a simple example of a DFS encoding, protecting against collective dephasing. Section 4 shows how to perform universal QC on this encoding. We then briefly and formally review the dynamical decoupling method, in Section 5. The methods are then combined in Sections 6,7, where we show how BB pulses can eliminate decoherence sources beyond collective dephasing. In Section 8 we present a new and somewhat surprising result: the elimination of off-resonant transitions, that will typically be induced by BB pulses, via BB pulses. Section 9 shows how to combine all the pieces together, by including logic gates with the BB pulses. Concluding remarks are presented in section 10. 


\section{DECOHERENCE}

The dynamics of an open quantum system coupled to a bath is formally obtained from the time-ordered evolution

$$
U(t)=\mathcal{T} \exp \left(-i \int^{t} H\left(t^{\prime}\right) d t^{\prime}\right)
$$

under the combined system-bath Hamiltonian

$$
\begin{aligned}
H & =\left(H_{S}+H_{C}\right) \otimes I_{B}+I_{S} \otimes H_{B}+H_{S B} \\
H_{S B} & =\sum_{\gamma} S_{\gamma} \otimes B_{\gamma},
\end{aligned}
$$

where $I$ is the identity operator, $H_{S}\left(H_{B}\right)$ is the internal Hamiltonian for the system (bath) alone, $H_{C}$ is an externally applied control Hamiltonian, $H_{S B}$ is the system-bath interaction Hamiltonian, and the $S_{\gamma}\left(B_{\gamma}\right)$ are operators acting on the system (bath). The $S_{\gamma}$ play the role of error generators in QC. Making the standard assumption of initially decoupled system and bath, one traces over the bath degrees of freedom in order to obtain the time-evolved reduced system density matrix:

$$
\rho(t)=\operatorname{Tr}_{B}\left[U(t)\left(\rho(0) \otimes \rho_{B}(0)\right) U^{\dagger}(t)\right],
$$

where $\rho(0)\left[\rho_{B}(0)\right]$ is the initial density matrix of the system [bath]. As is well-known, $\rho(t)$ is in general a mixed state: $\operatorname{Tr}\left[\rho(t)^{2}\right]<1$, indicating that the environment has decohered the system (see, e.g., Ref. 48).

\section{SIMPLE EXAMPLE OF DECOHERENCE-FREE SUBSPACES}

We briefly review the simplest example of a DFS. Suppose that a system of $K$ qubits (two-level systems) is coupled to a bath in a symmetric way, and undergoes a dephasing process. This can be described by the system-bath Hamiltonian

$$
H_{S B}^{\text {coll-deph }}=\sum_{j=1}^{K} \sigma_{j}^{z} \otimes B^{z}
$$

where $\sigma_{j}^{z}$ is the Pauli- $z$ matrix acting on the $j$ th qubit and $B^{z}$ is an arbitrary bath operator. Under this interaction qubit $j$ undergoes the transformation

$$
|0\rangle_{j} \rightarrow|0\rangle_{j} \quad|1\rangle_{j} \rightarrow e^{i \phi}|1\rangle_{j}
$$

which $\left(\right.$ after $\operatorname{Tr}_{B}$ ) puts a random phase $\phi$ between the basis states $|0\rangle$ and $|1\rangle$ (eigenstates of $\sigma_{z}$ with respective eigenvalues +1 and -1$)$. This can also be described by the matrix $R_{z}(\phi)=\operatorname{diag}\left(1, e^{i \phi}\right)$ acting on the $\{|0\rangle,|1\rangle\}$ basis. As implied by (4), we assume that the phase has no space $(j)$ dependence, i.e., the dephasing process is invariant under qubit permutations. Since the errors can be expressed in terms of the single Pauli spin matrix $\sigma_{z}$ of the two-level system, this example is referred to as "collective phase damping", or "weak collective decoherence". ${ }^{47}$ The more general situation when errors involving all three Pauli matrices are present, i.e., dissipation and dephasing, is referred to as "strong collective decoherence" , ${ }^{47}$ or just "collective decoherence" ${ }^{22,24,26}$ Without encoding a qubit initially in an arbitrary pure state $|\psi\rangle_{j}=a|0\rangle_{j}+b|1\rangle_{j}$ will decohere. This can be seen by calculating its density matrix as an average over all possible values of $\phi$,

$$
\rho_{j}=\int_{-\infty}^{\infty} R_{z}(\phi)|\psi\rangle_{j}\langle\psi| R_{z}^{\dagger}(\phi) p(\phi) d \phi
$$

where $p(\phi)$ is a probability density, and we assume the initial state of all qubits to be a product state. For a Gaussian distribution, $p(\phi)=(4 \pi \alpha)^{-1 / 2} \exp \left(-\phi^{2} / 4 \alpha\right)$, it is simple to check that

$$
\rho_{j}=\left(\begin{array}{cc}
|a|^{2} & a b^{*} e^{-\alpha} \\
a^{*} b e^{-\alpha} & |b|^{2}
\end{array}\right) .
$$


The decay of the off-diagonal elements in the computational basis is a signature of decoherence.

Let us now consider what happens in the two-qubit Hilbert space. The four basis states undergo the transformation

$$
\begin{aligned}
& |0\rangle_{1} \otimes|0\rangle_{2} \rightarrow|0\rangle_{1} \otimes|0\rangle_{2} \\
& |0\rangle_{1} \otimes|1\rangle_{2} \rightarrow e^{i \phi}|0\rangle_{1} \otimes|1\rangle_{2} \\
& |1\rangle_{1} \otimes|0\rangle_{2} \rightarrow e^{i \phi}|1\rangle_{1} \otimes|0\rangle_{2} \\
& |1\rangle_{1} \otimes|1\rangle_{2} \rightarrow e^{2 i \phi}|1\rangle_{1} \otimes|1\rangle_{2} .
\end{aligned}
$$

Observe that the basis states $|0\rangle_{1} \otimes|1\rangle_{2}$ and $|1\rangle_{1} \otimes|0\rangle_{2}$ acquire the same phase. This suggests that a simple encoding trick can solve the decoherence problem. Let us define encoded states by $\left|0_{L}\right\rangle=|0\rangle_{1} \otimes|1\rangle_{2} \equiv|01\rangle$ and $\left|1_{L}\right\rangle=|10\rangle$. Then the state $\left|\psi_{L}\right\rangle=a\left|0_{L}\right\rangle+b\left|1_{L}\right\rangle$ evolves under the dephasing process as

$$
\left|\psi_{L}\right\rangle \rightarrow a|0\rangle_{1} \otimes e^{i \phi}|1\rangle_{2}+b e^{i \phi}|1\rangle_{1} \otimes|0\rangle_{2}=e^{i \phi}\left|\psi_{L}\right\rangle
$$

and the overall phase thus acquired is clearly unimportant. This means that the 2-dimensional subspace $D F S_{2}(0)=\operatorname{Span}\{|01\rangle,|10\rangle\}$ of the 4-dimensional Hilbert space of two qubits is decoherence-free (DF). The subspaces $D F S_{2}(2)=\operatorname{Span}\{|00\rangle\}$ and $D F S_{2}(-2)=\operatorname{Span}\{|11\rangle\}$ are also (trivially) DF, since they each acquire a global phase as well, 1 and $e^{2 i \phi}$ respectively. Since the phases acquired by the different subspaces differ, there is decoherence between the subspaces.

For $K=3$ qubits a similar calculation reveals that the subspaces $D F S_{3}(1)=\operatorname{Span}\{|001\rangle,|010\rangle,|100\rangle\}$ and $D F S_{3}(-1)=\operatorname{Span}\{|011\rangle,|101\rangle,|110\rangle\}$ are DF, as well the (trivial) subspaces $D F S_{3}(3)=\operatorname{Span}\{|000\rangle\}$ and $D F S_{3}(3)=\operatorname{Span}\{|111\rangle\}$.

More generally, let

$$
\lambda_{K}=\text { number of } 0 \text { 's minus the number of } 1 \text { 's }
$$

in a computational basis state (i.e., a bitstring) over $K$ qubits. Then it is easy to check that any subspace spanned by states with constant $\lambda_{K}$ is DF, and can be denoted $D F S_{K}\left(\lambda_{K}\right)$ in accordance with the notation above. The dimensions of these subspaces are given by the binomial coefficients: $d \equiv \operatorname{dim}\left[D F S_{K}\left(\lambda_{K}\right)\right]=\left(\begin{array}{c}K \\ \lambda_{K}\end{array}\right)$ and they each encode $\log _{2} d$ qubits.

The encoding for the "collective phase damping" model discussed here has been tested experimentally. The first-ever experimental implementation of DFSs used the $D F S_{2}(0)$ subspace to protect against artifially induced decoherence in a linear optics setting. ${ }^{49}$ The same encoding was subsequently used to alleviate the problem of external fluctuating magnetic fields in an ion trap quantum computing experiment, ${ }^{50}$ and figures prominently in theoretical constructions of encoded, universal QC. ${ }^{13,16,21}$

\section{ENCODED UNIVERSAL LOGIC GATES}

Next we show how to perform universal QC on the encoding $D F S_{2}(0)=\operatorname{Span}\{|01\rangle,|10\rangle\}$.

\subsection{Single encoded-qubit gates}

Let $X_{i}, Y_{i}, Z_{i}$ denote the standard Pauli matrices $\sigma_{i}^{x}, \sigma_{i}^{y}, \sigma_{i}^{z}$, acting on the $i$ th physical qubit (we will use both notations interchangeably). In Ref. 12 it was shown that for the code $\left\{\left|0_{L}\right\rangle=|01\rangle,\left|1_{L}\right\rangle=|10\rangle\right\}$ the encoded logical operations (involving the first two physical qubits) are

$$
\begin{aligned}
\bar{X}_{12} & =\frac{1}{2}\left(X_{1} X_{2}+Y_{1} Y_{2}\right), \\
\bar{Y}_{12} & =\frac{1}{2}\left(Y_{1} X_{2}-X_{1} Y_{2}\right), \\
\bar{Z}_{12} & =\frac{1}{2}\left(Z_{1}-Z_{2}\right) .
\end{aligned}
$$


These operations form an $s u(2)$ algebra (i.e., we think of them as Hamiltonians rather than unitary operators). We use a bar to denote logical operations on the encoded qubits. In Refs. ${ }^{12-14}$ these logical operations were denoted by $T_{12}^{\alpha}, \alpha \in\{x, y, z\}$, and a detailed analysis was given on how to use typical solid-state Hamiltonians (Heisenberg, $X X Z$, and $X Y$ models, defined below) to implement quantum logic operations using this DFS encoding. E.g., the term $X_{1} X_{2}+Y_{1} Y_{2}$ is the spin-spin interaction in the $X Y$ model, and $Z_{1}-Z_{2}$ represents a Zeeman splitting. A static Zeeman splitting and a controllable $X Y$ interaction can be used to generate a universal set of logic gates. Similar conclusions hold when the $X Y$ interaction is replaced by a Heisenberg ${ }^{13,51,52}$ or $X X Z$ interaction, ${ }^{14}$ or even by a Heisenberg interaction that includes an anistropic spin-orbit term. ${ }^{17} \mathrm{We}$ remark that, as first shown in Refs. 46, 47, the various types of exchange interactions can be made universal also without any single-qubit terms (such as a Zeeman splitting), by encoding into three or more qubits, ${ }^{47,53-56}$ a result that has been termed "encoded universality". ${ }^{57}$

The two-qubit gate can be expressed as follows:

$$
\begin{aligned}
U_{i j}\left(\theta, \phi_{i}, \phi_{j}\right) & \equiv \exp \left(i \theta X_{\phi_{i}} X_{\phi_{j}}\right) \\
& =\cos \theta I_{i} I_{j}+i \sin \theta X_{\phi_{i}} X_{\phi_{j}}
\end{aligned}
$$

where

$$
X_{\phi} \equiv X \cos \phi+Y \sin \phi
$$

and $I$ is the identity operator. In the context of trapped-ion QC, ${ }^{58}$ the phase $\phi_{i}$ is the phase of the driving laser at the $i$ th qubit, while $\theta$ is proportional to the Rabi frequency, and can be set over a wide range of values. ${ }^{59}$ Introducing the operators

$$
\tilde{X}_{i j} \equiv \frac{1}{2}\left(X_{i} X_{j}-Y_{i} Y_{j}\right), \quad \tilde{Y}_{i j} \equiv \frac{1}{2}\left(Y_{i} X_{j}+X_{i} Y_{j}\right)
$$

(denoted $R_{i j}^{x}, R_{i j}^{y}$ respectively in Refs. 12-14) we can express

$$
U_{i j}\left(\theta, \phi_{i}, \phi_{j}\right)=\cos \theta \bar{I}+i \sin \theta\left(\cos \Delta \phi_{i j} \bar{X}_{i j}+\sin \Delta \phi_{i j} \bar{Y}_{i j}+\cos \Phi_{i j} \tilde{X}_{i j}+\sin \Phi_{i j} \tilde{Y}_{i j}\right),
$$

where $\Phi_{i j}=\phi_{i}+\phi_{j}$. It is simple to check that $\tilde{X}_{i j}$ and $\tilde{Y}_{i j}$ annihilate the code subspace $\left\{\left|0_{L}\right\rangle=|01\rangle,\left|1_{L}\right\rangle=|10\rangle\right\}$ and have non-trivial action (as encoded $X$ and $Y$ ) on the orthogonal subspace $\{|00\rangle,|11\rangle\}$. Therefore, upon restriction to the DFS we can write:

$$
\begin{aligned}
U_{i j}\left(\theta, \phi_{i}, \phi_{j}\right) & \stackrel{\text { DFS }}{\mapsto} \bar{U}_{i j}\left(\theta, \Delta \phi_{i j}\right) \\
& =\exp \left(i \theta \bar{X}_{\Delta \phi_{i j}}\right)=\cos \theta \bar{I}+i \sin \theta \bar{X}_{\Delta \phi_{i j}} .
\end{aligned}
$$

The fact that $\bar{U}_{i j}$ depends only on the relative phase $\Delta \phi_{i j}$ is crucial in the trapped-ion context: this quantity can be controlled by adjusting the angle between the driving laser and the interatomic axis, as well as by small adjustments of the trap voltages (which, in turn, control the trap oscillation frequency, and hence the qubit spacing), whereas it is much harder to control the absolute phase $\phi_{i},{ }^{60,61}$ and hence also $\Phi_{i j}$. This is why the code subspace $\{|01\rangle,|10\rangle\}$ enjoys a preferred status over the subspace $\{|00\rangle,|11\rangle\}$.

The Hamiltonians in Eq. (14) generate the logic operations (19):

$$
\begin{aligned}
\exp \left(i \theta \bar{X}_{12}\right) & =U_{12}(\theta, \phi, \phi)=\bar{U}_{12}(\theta, 0) \\
\exp \left(i \theta \bar{Y}_{12}\right) & =U_{12}\left(\theta, \phi, \phi+\frac{\pi}{2}\right)=\bar{U}_{12}\left(\theta, \frac{\pi}{2}\right) \\
\exp \left(i \theta \bar{Z}_{12}\right) & =\exp \left(i \frac{\pi}{4} \bar{Y}_{12}\right) \exp \left(i \theta \bar{X}_{12}\right) \exp \left(-i \frac{\pi}{4} \bar{Y}_{12}\right) \\
& =\bar{U}_{12}\left(\frac{\pi}{4}, \pi / 2\right) \bar{U}_{12}(\theta, 0) \bar{U}_{12}\left(-\frac{\pi}{4}, \pi / 2\right) .
\end{aligned}
$$

The third line follows from the elementary operator identity

$$
X_{\phi}=X \cos \phi+Y \sin \phi=e^{-i \phi Z / 2} X e^{i \phi Z / 2}
$$

which holds for any $s u(2)$ angular momentum set $\{X, Y, Z\}$, i.e., operators that satisfy the commutation relation $[X, Y]=2 i Z$ (and cyclic permutations thereof), in particular also the encoded operators $\{\bar{X}, \bar{Y}, \bar{Z}\}$. 


\subsection{Entangling gate between pairs of encoded qubits}

It is a well-known requirement of $\mathrm{QC}$ that in order to enact a universal set of logic gates (that allow any unitary transformation to be implemented) it is suffient to be able to implement all single-qubit operations (as in the previous subsection), and to entangle pairs of qubits. ${ }^{62}$ In this subsection we discuss the latter requirement. ions:

In Ref. 61 the following unitary gate was introduced, which is particularly suitable for QC using trapped

$$
\begin{aligned}
U_{4}= & \exp \left(-i \frac{\pi}{4} X_{\phi_{1}} X_{\phi_{2}} X_{\phi_{3}} X_{\phi_{4}}\right) \\
= & \frac{1}{\sqrt{2}}\left(I_{1} I_{2} I_{3} I_{4}-i X_{\phi_{1}} X_{\phi_{2}} X_{\phi_{3}} X_{\phi_{4}}\right) \\
& \stackrel{\operatorname{DFS}}{\mapsto} \frac{1}{\sqrt{2}}\left(\bar{I}_{12} \bar{I}_{34}-i \bar{X}_{\Delta \phi_{12}} \bar{X}_{\Delta \phi_{34}}\right) \\
= & \exp \left(-i \frac{\pi}{4} \bar{X}_{\Delta \phi_{12}} \bar{X}_{\Delta \phi_{34}}\right) .
\end{aligned}
$$

This gate can be used to entangle two DFS-qubits. It involves simultaneous control over two phase differences $\Delta \phi_{12}, \Delta \phi_{34}$, and thus control over the motion of two pairs of qubits. The case $\Delta \phi_{12}=\Delta \phi_{34}=0$ was used in Ref. 60 to demonstrate entanglement of four trapped-qubit qubits, but this choice is not unique.

For general exchange Hamiltonians a different method is required. We review the encoded recoupling method introduced in Ref. 13. The exchange interaction quite generally has the form

$$
\begin{aligned}
H_{\mathrm{ex}} & =\sum_{\alpha=x, y, z} \sum_{i<j} J_{i j}^{\alpha} \sigma_{i}^{\alpha} \sigma_{j}^{\alpha} \\
& =\sum_{i<j} J_{i j}^{-} R_{i j}^{x}+J_{i j}^{+} T_{i j}^{x}+J_{i j}^{z} \sigma_{i}^{z} \sigma_{j}^{z},
\end{aligned}
$$

where

$$
T_{i j}^{x}=\frac{1}{2}\left(\sigma_{i}^{x} \sigma_{j}^{x}+\sigma_{i}^{y} \sigma_{j}^{y}\right), \quad R_{i j}^{x}=\frac{1}{2}\left(\sigma_{i}^{x} \sigma_{j}^{x}-\sigma_{i}^{y} \sigma_{j}^{y}\right),
$$

and $J_{i j}^{ \pm}=J_{i j}^{x} \pm J_{i j}^{y}$.

The isotropic (Heisenberg) case corresponds to $J_{i j}^{\alpha} \equiv J_{i j}$. The $X Y$ model is the case $J_{i j}^{x}=J_{i j}^{y}, J_{i j}^{z}=0$. The $X X Z$ model is the case $J_{i j}^{x}= \pm J_{i j}^{y} \neq J_{i j}^{z}$. A summary of QC proposals that fall into each category can be found in Ref. 13. The free Hamiltonian is

$$
H_{0}=\sum_{i} \frac{1}{2} \varepsilon_{i} \sigma_{i}^{z}
$$

where $\varepsilon_{i}$ is the single-particle spectrum. In each instance of $H_{\mathrm{ex}}$ (Heisenberg, $X Y, X X Z$ ) one typically has control over only one type of parameter out of the set $\left\{J_{i j}^{\alpha}, \varepsilon_{i}\right\}$.

Let $J_{m}^{\alpha} \equiv J_{2 m-1,2 m}^{\alpha}(\alpha=z, \pm)$, and $\epsilon_{m}^{ \pm} \equiv\left(\varepsilon_{2 m-1} \pm \varepsilon_{2 m}\right) / 2$. Let $A$ and $B$ be two angular momentum operators satisfying $s u(2)$ commutation relations. Then if follows from Eq. (21) that the operation of "conjugating by $A "$,

$$
\begin{aligned}
C_{A} \circ \exp (i B) & \equiv \exp (-i A \pi / 2) \exp (i B) \exp (i A \pi / 2) \\
& =\exp (-i B)
\end{aligned}
$$

causes $B$ 's sign to be flipped.

Consider now an $X X Z$-type Hamiltonian where the $J_{m}^{+}$parameters are controllable but $\epsilon_{m}^{-}$and $J_{m}^{z}$ are fixed. For simplicity let us consider just the case $J_{m}^{-}=0$. Then we can rewrite

$$
H=H_{0}+H_{\mathrm{ex}}=\sum_{m=1}^{N / 2} \epsilon_{m}^{-} T_{m}^{z}-J_{m}^{z} T_{m}^{z} T_{m+1}^{z}+J_{m}^{+} T_{m}^{x},
$$


where we have omitted a constant term. The important point is now that $T_{m}^{x}$ and $T_{m}^{z}$ satisfy $s u(2)$ commutation relations. Therefore by using recoupling through "conjugation by $T_{m}^{x}$ " we can selectively turn on and off the single-encoded-qubit rotation $T_{m}^{z}$ and the encoded-Ising interaction $T_{m}^{z} T_{m+1}^{z}$. This example of "encoded selective recoupling" establishes that encoded universal computation in the $X X Z$ model can be done using control over the $J_{m}^{+}$parameters alone.

Next, consider the $X Y$ model, with controllable $J_{i j}^{+}$, but fixed $\varepsilon_{i}$. To implement encoded single-qubit operations, we can use the same encoded recoupling method as for the $X X Z$ model. As for encoded two-qubit operations, we now no longer have the $\sigma_{i}^{z} \sigma_{j}^{z}$ terms. Since the $X Y$ model with nearest-neighbor interactions can be shown not to be universal, ${ }^{14}$ we turn on also next-nearest neighbor $J_{i j}^{+}$terms (these can still be nearestneighbor in a $2 \mathrm{D}$ hexagonal geometry). First note that $C_{T_{12}^{x}} \circ T_{23}^{x}=i \sigma_{1}^{z} \sigma_{2}^{z} T_{13}^{x}$. Now assume we can control $J_{13}^{+}$; then, using conjugation by $\pi / 4: C_{\frac{1}{2} T_{13}^{x}} \circ\left(C_{T_{12}^{x}} \circ T_{23}^{x}\right)=\sigma_{2}^{z}\left(\sigma_{3}^{z}-\sigma_{1}^{z}\right) / 2$. Since $\sigma_{1}^{z} \sigma_{2}^{z}$ is constant on the code subspace it can be ignored. On the other hand, $\sigma_{2}^{z} \sigma_{3}^{z}$ again acts as $-T_{1}^{z} T_{2}^{z}$, i.e., as an encoded $\sigma^{z} \otimes \sigma^{z}$. This establishes universal encoded computation in the $X Y$ model.

Taken together, the results in this section show how universal QC can be implemented using qubits encoded into a DFS offering protection against collective dephasing, while using only reasonable models of physically controllable Hamiltonians. We now move on to a discussion of how to reduce additional sources of decoherence.

\section{DYNAMICAL DECOUPLING PULSES}

We briefly review the dynamical decoupling method, introduced in Ref. 27, and further developed in Refs. 15, 18, 19,21,28-45. A set of "symmetrization" operations is chosen such that they form a discrete subgroup of the full unitary group of operations on the Hilbert space of the system. Denote this group $\mathcal{G}=\left\{V_{j}\right\}_{j=1}^{|\mathcal{G}|}$ where $V_{1}=I$. The cycle time is $T_{c}=|\mathcal{G}| \Delta t$, where $|\mathcal{G}|$ is the number of symmetrization operations, and $\Delta t$ is the time that the system evolves freely between operations under $U_{0}(t) \equiv \exp \left(-i H_{S} t\right)$. The symmetrized evolution after a single cycle is given by

$$
U\left(T_{c}\right)=\prod_{j=1}^{|\mathcal{G}|} V_{j}^{\dagger} U_{0}(\Delta t) V_{j} \equiv e^{i H_{\mathrm{eff}} T_{c}},
$$

where the evolution under $H_{S B}+H_{B}$ has been neglected during application of the pulses $V_{j}$, which quantifies the sense in which these pulses have to be fast and strong (for details see, e.g, Refs. 21,27,30,44,45). $H_{\text {eff }}$ denotes the resulting effective Hamiltonian, that can be computed to any order using the Magnus expansion. ${ }^{63}$ In the BB limit one is interested in the evolution $U\left(N T_{c}\right)$ after $N$ cycles, such that $N \rightarrow \infty$ and $\Delta t \rightarrow 0$ while $N \Delta t$ is finite. As shown in Ref. 34 one can then retain only the first order Magnus term, approximating $H_{\text {eff }}$ by

$$
H_{\text {eff }}=\frac{1}{|\mathcal{G}|} \sum_{j=1}^{|\mathcal{G}|} V_{j}^{\dagger} H V_{j} \equiv \Pi_{\mathcal{G}}(H) .
$$

The map $\Pi_{\mathcal{G}}$ is the projector into the centralizer $Z$ of $\mathcal{G}$, defined as

$$
Z(\mathcal{G})=\left\{X \mid V_{j}^{\dagger} X V_{j}=X, \forall V_{j} \in \mathcal{G}\right\}
$$

Since the pulses $g_{j}$ are unitary the centralizer equals the commutant in this case:

$$
Z(\mathcal{G})=\left\{X \mid\left[X, V_{j}\right]=0, \forall V_{j} \in \mathcal{G}\right\} .
$$

It is clear that $\Pi_{\mathcal{G}}$ commutes with the adjoint action $V_{j} \cdot V_{j}^{\dagger}$ for all $j$, so that if $\mathcal{G}$ is generated by $\left\{I, H_{S}, S_{\gamma}\right\}$, the evolution will proceed without the operators $S_{\gamma}$ affecting the system as errors: the evolution has been symmetrized with respect to $\mathcal{G} .{ }^{31}$ For a geometric interpretation of this symmetrization see Ref. 41, where it was also pointed out that while the $\mathrm{BB}$ pulses should have the effect of rotating $H$ to the vertices of a symmetric object, they need in fact not form a group. 


\section{CREATING COLLECTIVE DEPHASING CONDITIONS USING DECOUPLING PULSES: REDUCING DECOHERENCE DURING STORAGE}

One of the important advantages of the DFS encoding $\{|01\rangle,|10\rangle\}$ is that it is immune to collective dephasing. However, other sources of decoherence inevitably remain. In this and the following section, we algebraically classify all additional decoherence effects and show how they can be eliminated.

\subsection{Creating collective dephasing on a pair of qubits}

First, let us analyze the effect of breaking the collective dephasing symmetry, by considering a system-bath interaction of the form

$$
H_{S B}^{\mathrm{deph}(2)}=Z_{1} \otimes B_{1}^{z}+Z_{2} \otimes B_{2}^{z}
$$

where $B_{1}^{z}, B_{2}^{z}$ are arbitrary bath operators [compare to Eq. (4)]. This describes a general dephasing interaction on two qubits. The source of such dephasing during storage can be long wavelength, randomly fluctuating ambient magnetic fields, ${ }^{50}$ that randomly shift the relative phase between the qubit $|0\rangle$ and $|1\rangle$ states through the Zeeman effect. The interaction can be rewritten as a sum over a collective dephasing term $Z_{1}+Z_{2}$ and another, differential dephasing term $Z_{1}-Z_{2}$, that is responsible for errors on the DFS:

$$
H_{S B}^{\mathrm{deph}(2)}=\left(Z_{1}+Z_{2}\right) \otimes B_{\mathrm{col}}^{z}+\left(Z_{1}-Z_{2}\right) \otimes B_{\mathrm{dif}}^{z} .
$$

Here $B_{\text {col }}^{z}=\left(B_{1}^{z}+B_{2}^{z}\right) / 2$ and $B_{\mathrm{dif}}^{z}=\left(B_{1}^{z}-B_{2}^{z}\right) / 2$. If $B_{\mathrm{dif}}^{z}$ were zero then there would only be collective dephasing and the DFS encoding would offer perfect protection. However, in general $B_{\mathrm{dif}}^{z} \neq 0$, and the DFS encoding will not suffice to offer complete protection.

The crucial observation is that, since $Z_{1}-Z_{2} \propto \bar{Z}_{12}$ [recall Eq. (14)], the offending term causes logical errors on the DFS. ${ }^{16}$ As shown in Refs. 15,37, then the problem of $B_{\text {dif }}^{z} \neq 0$ can be solved using a series of pulses that symmetrize $H_{S B}^{\operatorname{deph}(2)}$ such that only the collective term remains. To do so note that since the offending term $\propto \bar{Z}_{12}$, it anticommutes with $\bar{X}_{12}=\frac{1}{2}\left(X_{1} X_{2}+Y_{1} Y_{2}\right)$. At the same time $\bar{X}_{12}$ commutes with $Z_{1}+Z_{2}$. This allows us to flip the sign of the offending term by using a pair of $\pm \pi / 2$ pulses in $\bar{X}_{12}$, while leaving only the collective term. Evolution with the flipped sign followed by unaltered evolution leads to cancellation of the offending term. Specifically: ${ }^{15}$

$$
e^{-i H_{S B} \tau} e^{-i \frac{\pi}{2} \bar{X}_{12}} e^{-i H_{S B} \tau} e^{i \frac{\pi}{2} \bar{X}_{12}}=e^{-i\left(Z_{1}+Z_{2}\right) \otimes B_{\mathrm{col}}^{z} 2 \tau},
$$

or, in terms of gates:

$$
e^{-i H_{S B} \tau} \bar{U}_{12}\left(-\frac{\pi}{2}, 0\right) e^{-i H_{S B} \tau} \bar{U}_{12}\left(\frac{\pi}{2}, 0\right)=e^{-i\left(Z_{1}+Z_{2}\right) \otimes B_{\mathrm{col}}^{z} 2 \tau},
$$

where $\bar{U}_{i j}\left(\theta, \Delta \phi_{i j}\right)$ was defined in Eq. (19). This equation means that the system-bath coupling effectively looks like collective dephasing at the end of the pulse sequence. Thus, the system is periodically (every $2 \tau$ ) projected into the DFS.

In order for the the procedure described in Eq. (33) to work, the gate $\bar{U}_{12}\left( \pm \frac{\pi}{2}, 0\right)$ must be executed at a timescale faster than the cutoff frequency associated with the fluctuating (magnetic) fields causing the differential dephasing term in $H_{S B}^{\operatorname{deph}(2)}$.

\subsection{Creating collective dephasing on a block of four qubits}

So far we have discussed creation of collective dephasing conditions on a single DFS qubit. However, it is essential for the reliable execution of an entangling logic gate to have collective dephasing over all four qubits participating in the gate, even if only two are coupled at a time. A procedure for creating collective decoherence conditions over blocks of 3,4,6 and 8 qubits was given in Ref. 15. Here we show how to do the same for a block of 4 qubits with collective dephasing. 
Let us start with a general dephasing Hamiltonian on $N$ qubits, and rewrite it in terms of nearest-neighbor sums and differences:

$$
\begin{aligned}
H_{S B}^{\mathrm{deph}} & =\sum_{i=1}^{N} Z_{i} \otimes B_{i} \\
& =\sum_{j=1}^{N / 2}\left(Z_{2 j}+Z_{2 j-1}\right) \otimes B_{2 j}^{+}+\left(Z_{2 j}-Z_{2 j-1}\right) \otimes B_{2 j}^{-},
\end{aligned}
$$

where $B_{2 j}^{ \pm} \equiv\left(B_{2 j} \pm B_{2 j-1}\right) / 2$. As noted above, $Z_{2 j}-Z_{2 j-1} \propto \bar{Z}_{2 j-1,2 j}$, so that to eliminate all nearest-neighbor differences of the form $\left(Z_{2 j}-Z_{2 j-1}\right)$ we can use the collective decoupling pulse $X_{n n}=\bigotimes_{j=1}^{N / 2} e^{i \frac{\pi}{2} \bar{X}_{2 j-1,2 j}}$ :

$$
e^{-i H_{S B} \tau} X_{n n} e^{-i H_{S B} \tau} X_{n n}^{\dagger}=e^{-i 2 \tau \sum_{j=1}^{N / 2}\left(Z_{2 j}+Z_{2 j-1}\right) \otimes B_{2 j}^{+}},
$$

or, in gate terms:

$$
e^{-i H_{S B} \tau}\left[\bigotimes_{j=1}^{N / 2} \bar{U}_{2 j-1,2 j}\left(-\frac{\pi}{2}, 0\right)\right] e^{-i H_{S B} \tau}\left[\bigotimes_{j=1}^{N / 2} \bar{U}_{2 j-1,2 j}\left(\frac{\pi}{2}, 0\right)\right]=e^{-i 2 \tau \sum_{j=1}^{N / 2}\left(Z_{2 j}+Z_{2 j-1}\right) \otimes B_{2 j}^{+}}
$$

The next step is to eliminate next-nearest neighbor differential terms. To this end let us rewrite the outcome of the $X_{n n}$ pulse in terms of sums and differences over blocks of four ions:

$$
\begin{aligned}
\sum_{j=1}^{N / 2}\left(Z_{2 j}+Z_{2 j-1}\right) \otimes B_{2 j}^{+} & =\sum_{j=1}^{N / 2}\left[Z_{2 j+2}+Z_{2 j+1}+Z_{2 j}+Z_{2 j-1}\right] \otimes B_{2 j}^{+,+} \\
& +\sum_{j=1}^{N / 2}\left[\left(Z_{2 j+2}-Z_{2 j}\right)+\left(Z_{2 j+1}-Z_{2 j-1}\right)\right] \otimes B_{2 j}^{+,-},
\end{aligned}
$$

where $B_{2 j}^{+, \pm} \equiv\left(B_{2 j+2}^{+} \pm B_{2 j}^{+}\right) / 2$. The term in the first line contains only the desired block-collective dephasing over 4 ions. The term in the second line contains undesired differential dephasing terms that we wish to eliminate. But these terms once again have the appearance of encoded $Z$ operators, between next-nearest neighbor ion pairs. Therefore we need to apply a second collective pulse $X_{n n n}=\bigotimes_{j=1}^{N / 2} e^{i \frac{\pi}{2} \bar{X}_{2 j-1,2 j+1}} e^{i \frac{\pi}{2} \bar{X}_{2 j, 2 j+2}}$, that applies encoded $X$ operators on these qubit pairs. At this point we are left just with collective dephasing terms on blocks of 4 qubits, as required:

$$
\begin{aligned}
& e^{-i 2 \tau \sum_{j=1}^{N / 2}\left(Z_{2 j}+Z_{2 j-1}\right) \otimes B_{2 j}^{+}}\left[\bigotimes_{j=1}^{N / 2} \bar{U}_{2 j-1,2 j+1}\left(-\frac{\pi}{2}, 0\right) \bar{U}_{2 j, 2 j+2}\left(-\frac{\pi}{2}, 0\right)\right] \times \times \\
& e^{-i 2 \tau \sum_{j=1}^{N / 2}\left(Z_{2 j}+Z_{2 j-1}\right) \otimes B_{2 j}^{+}}\left[\bigotimes_{j=1}^{N / 2} \bar{U}_{2 j-1,2 j+1}\left(\frac{\pi}{2}, 0\right) \bar{U}_{2 j, 2 j+2}\left(\frac{\pi}{2}, 0\right)\right]=e^{-i 4 \tau \sum_{j=1}^{N / 2}\left(Z_{2 j+2}+Z_{2 j+1}+Z_{2 j}+Z_{2 j-1}\right) \otimes B_{2 j}^{++}} .
\end{aligned}
$$

This pulse sequence is important to ensure that collective dephasing conditions will prevail during the execution of logic gates between DFS qubits.

\section{REDUCTION OF ALL REMAINING DECOHERENCE ON A SINGLE DFS QUBIT DURING LOGIC GATE EXECUTION}

The reduction of differential dephasing errors, as in the previous subsection, is particularly relevant for storage errors. However, this is only the first step. Additional sources of decoherence may take place during storage, 
and in particular during the execution of logic gates. It is useful to provide a complete algebraic classification of the possible decoherence processes. This will allow us to see what can be done using decoupling pulses. To this end let us now write the system-bath Hamiltonian on two physical qubits in the general form

$$
H_{S B}=H_{\mathrm{Leak}}+H_{\mathrm{Logi}}+H_{\mathrm{DFS}}
$$

where

$$
\begin{aligned}
H_{\mathrm{DFS}} & =\operatorname{Span}\left\{\frac{Z I+I Z}{2}, \frac{X Y+Y X}{2}, \frac{X X-Y Y}{2}, Z Z, I I\right\} \\
H_{\text {Leak }} & =\operatorname{Span}\{X I, I X, Y I, I Y, X Z, Z X, Y Z, Z Y\} \\
H_{\text {Logi }} & =\operatorname{Span}\left\{\bar{X}=\frac{X X+Y Y}{2}, \bar{Y}=\frac{Y X-X Y}{2}, \bar{Z}=\frac{Z I-I Z}{2}\right\}
\end{aligned}
$$

where $I$ is the identity operator, $X Z \equiv X_{1} Z_{2}$ (etc.), and where Span means a linear combination of these operators tensored with bath operators. The 16 operators in Eq. (40) form a complete basis for all 2-qubit operators. This classification, first introduced in Ref. 16, has the following significance. The operators in $H_{\mathrm{DFS}}$ either vanish on the DFS, or are proportional to identity on it. In either case their effect is to generate an overall phase on the DFS, so they can be safely ignored from now on. The operators in $H_{\text {Leak }}$ are the leakage errors: terms that cause transitions between states inside and outside of the DFS. A universal and efficient decoupling method for eliminating such errors, for arbitrary numbers of (encoded) qubits was given in Ref. 18. Finally, the operators in $H_{\text {Logi }}$ have the form of logic gates on the DFS. However, these are undesired logic operations, since they are coupled to the bath, and thus cause decoherence.

In the previous subsection we showed how to eliminate the logical error $\bar{Z}$, but we see now that this was only one error in a much larger set. To deal with the additional errors it is useful at this point to introduce a more compact notation for the pulse sequences. We denote by $[\tau]$ a period of evolution under the free Hamiltonian, i.e., $U(\tau) \equiv \exp \left(-i H_{S B} \tau\right) \equiv[\tau]$, and further denote

$$
P \equiv \bar{U}_{12}\left(-\frac{\pi}{2}, 0\right)=\exp \left(-i \frac{\pi}{2} \bar{X}_{12}\right)
$$

Thus Eq. (33) can be written as:

$$
\exp \left[-i\left(B_{1}^{z}+B_{2}^{z}\right)\left(Z_{1}+Z_{2}\right) \tau\right]=\left[\tau, P, \tau, P^{\dagger}\right] .
$$

As a first step in dealing with the additional errors, note that the symmetrization procedure $\left[\tau, P, \tau, P^{\dagger}\right]$ can in fact achieve more than just the elimination of the differential dephasing $Z_{1}-Z_{2}$ term. Since $\bar{X}_{12}$ also anticommutes with $\bar{Y}_{12}=\frac{1}{2}\left(Y_{1} X_{2}-X_{1} Y_{2}\right) \in H_{\text {Logi }}$, if such a term appears in the system-bath interaction it too will be eliminated using the same procedure.

So far we have used a $\frac{\pi}{2} \bar{X}_{12}$ pulse. Interestingly, the Hamiltonian $\bar{X}_{12}$ can also be used to eliminate all leakage errors. ${ }^{16}$ To see this, note that $\bar{U}_{12}( \pm \pi, 0)=\exp \left( \pm i \pi \bar{X}_{12}\right)=Z_{1} Z_{2}$. This operator anticommutes with all terms in $H_{\text {Leak }}$. Hence it too can be used in a parity-kick pulse sequence, that will eliminate all the leakage errors.

At this point we are left with just a single error: $\bar{X}_{12} \otimes B$ itself, in $H_{\text {Logi }}$. Clearly, we cannot use a pulse generated by $\bar{X}_{12}$ to eliminate this error. Instead, to deal with this error we need to introduce one more pulse pair that anticommutes with $\bar{X}_{12}$, e.g., $\exp \left( \pm i \frac{\pi}{2} \bar{Y}_{12}\right)=\bar{U}_{12}\left( \pm \frac{\pi}{2}, \frac{\pi}{2}\right)$.

Let us now see how to combine all the decoherence elimination pulses into one efficient sequence. First we introduce the abbreviations

$$
\begin{aligned}
\Pi & \equiv \bar{U}_{12}( \pm \pi, 0)=\exp \left( \pm i \pi \bar{X}_{12}\right)=\Pi^{\dagger}=P P \\
Q & \equiv \bar{U}_{12}\left(-\frac{\pi}{2}, \frac{\pi}{2}\right)=\exp \left(-i \frac{\pi}{2} \bar{Y}_{12}\right) \\
\Lambda & \equiv \bar{U}_{12}\left( \pm \pi, \frac{\pi}{2}\right)=\exp \left( \pm i \pi \bar{Y}_{12}\right)=\Lambda^{\dagger}=Q Q
\end{aligned}
$$


As argued above, the $\pi$ pulse $\Pi$ eliminates $H_{\text {Leak }}$ :

$$
\exp \left[-i\left(H_{\mathrm{Logi}}+H_{\mathrm{DFS}}\right) 2 \tau\right]=[\tau, \Pi, \tau, \Pi] .
$$

Now let us discuss adding the extra pulses needed to achieve full decoherence elimination. The $\pi / 2$ pulse $P$ eliminates $\bar{Y}$ and $\bar{Z}$ in $H_{\text {Logi }}$. Combining this with the sequence for leakage elimination we have the sequence of 4 pulses:

$$
\begin{aligned}
e^{-i\left(H_{\mathrm{DFS}}+\bar{X} \otimes B_{\bar{X}}\right) 4 \tau} & =[U(\tau) \Pi U(\tau) \Pi] P^{\dagger}[U(\tau) \Pi U(\tau) \Pi] P \\
& =\left[\tau, \Pi, \tau, P, \tau, \Pi, \tau, P^{\dagger}\right]
\end{aligned}
$$

(where we have used $\Pi P^{\dagger}=P, \Pi P=P^{\dagger}$ ).

If we wish to entirely eliminate decoherence then we are left just with getting rid of the logical error due to $\bar{X}$. To eliminate it we now combine with the $\bar{Y}$-direction, $\pi / 2$ pulse, $Q$ :

$$
\begin{aligned}
e^{-i H_{\mathrm{DFS}} 8 \tau} & =\left[U(\tau) \Pi U(\tau) P U(\tau) \Pi U(\tau) P^{\dagger}\right] Q^{\dagger}\left[U(\tau) \Pi U(\tau) P U(\tau) \Pi U(\tau) P^{\dagger}\right] Q \\
& =\left[\tau, \Pi, \tau, P, \tau, \Pi, \tau, P^{\dagger}, Q^{\dagger}, \tau, \Pi, \tau, P, \tau, \Pi, \tau, P^{\dagger}, Q\right]
\end{aligned}
$$

which takes ten pulses. Unfortunately it is not possible to compress this further, since $P^{\dagger} Q=(i \bar{X})(-i \bar{Y})=$ $i \bar{Z}$ and $P^{\dagger} Q^{\dagger}=-i \bar{Z}$, neither of which cannot be generated directly (in one step) from the available gate $\bar{U}_{i j}\left(\theta, \Delta \phi_{i j}\right)=\cos \theta \bar{I}+i \sin \theta \bar{X}_{\Delta \phi_{i j}}$.

\section{ELIMINATION OF OFF-RESONANT TRANSITIONS}

One important caveat in our discussion so far is that, because we need very strong and fast pulses, our gate operation may become imperfect. Specifically, off-resonant coupling may become important. This can cause unitary leakage errors from the DFS. These can in turn be reduced using the methods in Refs. 64,65 . Here we present an alternative and new method, that uses BB pulses generated in terms of the system Hamiltonian.

Consider an $N$-level system Hamiltonian

$$
H_{0}=\sum_{i=1}^{N} E_{i}|i\rangle\langle i|
$$

where the levels $i=1,2$ denote our qubit. If all parameters are fixed, the evolution $U_{0}(t)=\exp \left(-i H_{0} t\right)$ is always on. If we turn on an interaction $H_{I}$, then the total Hamiltonian is $H=H_{0}+H_{I}$, which can be written as

$$
H=\sum_{i=1}^{N} H_{i i}|i\rangle\langle i|+\sum_{i>j>2}^{N}\left(H_{i j}|i\rangle\langle j|+\text { h.c. }\right)+H_{L}
$$

where the leakage term is

$$
H_{L}=|1\rangle \sum_{i=3}^{N} H_{1 i}\langle i|+| 2\rangle \sum_{i=3}^{N} H_{2 i}\langle i|+\text { h.c. }
$$

Now note that

$$
U_{0}^{\dagger}(t) H U_{0}(t)=\sum_{i=1}^{N} H_{i i}|i\rangle\langle i|+\sum_{i>j=1}^{N}\left(e^{-i\left(E_{i}-E_{j}\right) t} H_{i j}|i\rangle\langle j|+\text { h.c. }\right) .
$$

Using this we now show how to eliminate each of the leakage terms one by one. First, we eliminate $|1\rangle H_{13}\langle 3|+$ h.c. by the BB sequence

$$
2 H^{\prime}=H+U_{0}^{\dagger}\left(\frac{\pi}{E_{1}-E_{3}}\right) H U_{0}\left(\frac{\pi}{E_{1}-E_{3}}\right)
$$


The new Hamiltonian $H^{\prime}$ does not contain $|1\rangle H_{13}\langle 3|+$ h.c. Next, we eliminate $|2\rangle H_{23}^{\prime}\langle 3|+$ h.c. by $U_{0}\left(\frac{\pi}{E_{2}-E_{3}}\right)$, where $H_{23}^{\prime}=\exp \left(-i \frac{\pi\left(E_{2}-E_{3}\right)}{E_{1}-E_{3}}\right) H_{23}$. We can clearly repeat this procedure so as to eliminate all leakage. The crucial point is that since we have used the system Hamiltonian to generate BB pulses, off-resonant transitions do not take place: $H_{0}$ has no matrix elements between different levels.

A concern is how we to obtain $U_{0}^{\dagger}(t)=\exp \left(+i H_{0} t\right)$. This problem is shared by other methods dealing with the same problem. ${ }^{65}$ In principle it can be solved provided the level spacings are rationally related. Note in this context that usually we do not need to eliminate all $H_{1 i}$, since $H_{1 i} \gg H_{1 i+1}$ and typically decrease exponentially according to time-independent perturbation theory. We have assumed that there is no degeneracy. If there is, then all degenerate transitions will be eliminated simultaneously, so the procedure is simplified.

\section{COMBINING LOGIC GATES WITH DECOUPLING PULSES}

So far we have discussed computation using the encoded recoupling method (Section 4), and encoded decoupling (Sections 6,7). We now put the two together in order to obtain the full ERD picture. At least two methods are available for combining quantum computing operations with the sequences of decoupling pulses we have presented above. For a general analysis of this issue see Ref. 35 .

\subsection{Fast + Strong Gates Method}

The decoupling pulse sequences given in Sec. 7 "stroboscopically" create collective dephasing conditions at the conclusion of each cycle. As noted above, this is equivalent to a periodic projection into the DFS. This property allows for "stroboscopic" quantum computation at the corresponding projection times. ${ }^{35}$ Here the computation pulses need to be synchronized with the decoupling pulses, and inserted at the end of each cycle. Because of the conditions on the validity of the BB method, ${ }^{27}$ the amount of time available for implementation of a logic gate is no more than the bath correlation time $\tau_{c}=2 \pi / \omega_{c}$. (An exception to this rule is the case of $1 / f$ noise. ${ }^{44}$ ) Assuming the dominant decoherence contributions not accounted for by the DFS encoding to come from differential dephasing (setting the $\tau_{c}$ time-scale), and given that we already assumed that we can use pulses with interval $\Delta t \ll \tau_{c}$, it is consistent to assume that we can then also perform logic gates on the same time scale.

\subsection{Fast + Weak Gates Method}

There may be an advantage to using fast but weak pulses for the logic gates, while preserving the fast + strong property of the decoupling pulses. To see how to combine logic gates with decoupling in this case, let us denote by $H_{S}=X_{\phi_{i}} X_{\phi_{j}}$ the controllable system Hamiltonian that generates the entangling gate $U_{i j}\left(\theta, \phi_{i}, \phi_{j}\right)$ [recall Eq. (15)]. Suppose first that we turn on this logic-gate generating Hamiltonian in a manner that is neither very strong nor very fast, so that the system-bath interaction is not negligible while $H_{S}$ is on (this obviously puts less severe demands on experimental implementation). Then the corresponding unitary operator describing the dynamics of system plus bath is:

$$
\tilde{U}(t)=\exp \left[-i t\left(H_{S}+H_{S B}+H_{B}\right)\right] .
$$

Now, if we choose $H_{S}$ so that it commutes with the decoupling pulses, then we can show that after decoupling

$$
\tilde{U}(t) \mapsto \exp \left[-i 2 t\left(H_{S}+H_{B}\right)\right],
$$

provided $t$ is sufficiently small. Tracing out the bath then leaves a purely unitary, decoherence-free evolution on the system. To prove this, assume we have chosen $t^{\prime}$ and the decoupling Hamiltonian $H_{S}^{\prime}$ so that (i) $\exp \left(-i t^{\prime} H_{S}^{\prime}\right) H_{S B} \exp \left(i t^{\prime} H_{S}^{\prime}\right)=-H_{S B}$, and (ii) $\left[H_{S}^{\prime}, H_{S}\right]=0$. Then

$$
\begin{aligned}
& \tilde{U}(t) e^{-i t^{\prime} H_{S}^{\prime}} \tilde{U}(t) e^{-i t^{\prime} H_{S}^{\prime}}=\tilde{U}(t) e^{-i t\left[H_{S}+e^{-i t^{\prime} H_{S}^{\prime}} H_{S B} e^{i t^{\prime} H_{S}^{\prime}}+H_{B}\right]} \\
& =e^{-i t\left(H_{S}+H_{S B}+H_{B}\right)} e^{-i t\left(H_{S}-H_{S B}+H_{B}\right)} \\
& =e^{-\left\{2 i t\left(H_{S}+H_{B}\right)+t^{2}\left(\left[H_{S B}, H_{S}\right]+\left[H_{S B}, H_{B}\right]\right)+O\left(t^{3}\right)\right\}},
\end{aligned}
$$

where we have used the Baker-Campbell-Hausdorff formula, $\exp (\alpha A) \exp (\alpha B)=\exp \left\{\alpha(A+B)+\frac{\alpha^{2}}{2}[A, B]+\right.$ $\left.O\left(\alpha^{3}\right)\right\}$. 
Let us now show how to efficiently combine logic operations and decoupling pulses. For simplicity consider only the case where we can neglect the $\bar{X}$ error, i.e., our decoupling sequence is the 4-pulse one given in Eq. (45). Suppose we wish to implement a logical $X$ operation, i.e., $\exp \left(-i \theta \bar{X}_{12}\right)$. Recall that this involves turning on the Hamiltonian $H_{S}^{X}=\Omega_{X} X_{\phi} X_{\phi} \stackrel{\text { DFS }}{\mapsto} \Omega_{X} \bar{X}_{12}$ between two physical qubits. Because the decoupling pulses $P=\exp \left(-i \frac{\pi}{2} \bar{X}_{12}\right)$ and $\Pi=\exp \left( \pm i \pi \bar{X}_{12}\right)$ are generated in terms of the same Hamiltonian, they commute with $H_{S}^{X}$ while eliminating $H_{S B}$ (except for the terms in $H_{S B}$ that have trivial action on the DFS). Thus the conditions under which Eq. (48) were shown to hold are satisfied. This allows us to insert the logic gates into the four free evolution periods involved in the pulse sequence of Eq. (45). Thus, the full pulse sequence that combines creation of collective dephasing conditions with execution of the logic gate is:

$$
e^{-i t\left(\Omega_{X} \bar{X}_{12}+H_{\mathrm{DFS}}\right)}=\tilde{U}(t / 4) \Pi \tilde{U}(t / 4) P \tilde{U}(t / 4) \Pi \tilde{U}(t / 4) P^{\dagger}
$$

with $\tilde{U}(t)=\exp \left[-i t\left(H_{S}^{X}+H_{S B}+H_{B}\right)\right]$, and which, using the DFS encoding, is equivalent to the desired $\exp \left(-i \theta \bar{X}_{12}\right)$. This involves 8 control pulses, 4 of which are of the fast + strong type (those involving $P$ and $\Pi$ ), and 4 of which must be fast, but need not be so strong that we can neglect $H_{S B}$.

If we wish to implement logical $Y$ operation, i.e., $\exp \left(-i \theta \bar{Y}_{12}\right)$, then we cannot now use $P$ and $\Pi$, since they anticommute with $\bar{Y}_{12}$ and will eliminate it. Instead we should use decoupling pulses generated in terms of $\bar{Y}_{12}$, which will also have the desired effect of eliminating $H_{\text {Leak }}$, as well as $\bar{X}$ and $\bar{Z}$ logical errors, while commuting with the $\bar{Y}$ logic operations (and for this reason can of course not eliminate $\bar{Y}$ errors). These are just the $Q$ and $\Lambda$ pulses defined in Eq. (43). In gate terms this implies turning on the Hamiltonian $H_{S}^{Y}=\Omega_{Y} X_{\phi} X_{\phi+\pi / 2} \stackrel{\text { DFS }}{\mapsto} \Omega_{Y} \bar{Y}_{12}$ between two physical qubits. Thus:

$$
e^{-i t\left(\Omega_{Y} \bar{Y}_{12}+H_{\mathrm{DFS}}\right)}=\tilde{U}(t / 4) \Lambda \tilde{U}(t / 4) Q \tilde{U}(t / 4) \Lambda \tilde{U}(t / 4) Q^{\dagger}
$$

with $\tilde{U}(t)=\exp \left[-i t\left(H_{S}^{Y}+H_{S B}+H_{B}\right)\right]$, and which, using the DFS encoding, is equivalent to the desired $\exp \left(-i \theta \bar{Y}_{12}\right)$.

Finally, to generate single DFS-qubit rotations about an arbitrary axis we can combine Eqs. (52),(53) according to the Euler angles construction. ${ }^{62}$ Given that Eqs. (52),(53) each take 8 pulses, the Euler angle method will generate an arbitrary DFS-qubit rotation in at most 24 pulses.

Concerning gates that entangle two DFS-qubits, the situation may be more involved, since now the nextnearest neighbor pulses in Eq. (38), that create the collective dephasing conditions on four qubits, do not all commute with the $U_{4}$ gate of Eq. (22). Therefore here we must resort to the strong + fast method of the previous subsection, i.e., we need to synchronize the $U_{4}$ pulses with the end of the decoupling pulse sequence. We do not here analyze the situation with respect to the exchange Hamiltonian implementation of conditional logic gates.

Taken together, the methods described in this section provide an explicit way to implement universal QC in a manner that offers protection against all sources of qubit decoherence, using a fast + strong (or fast + weak) versions of logic gates.

\section{DISCUSSION AND CONCLUSIONS}

We have proposed a method of encoded recoupling and decoupling (ERD) for performing decoherence-protected quantum computation. Our method combines an encoding into qubit-pair decoherence-free subspaces (each pair yielding one encoded qubit), with sequences of recoupling and decoupling pulses. The qubit encoding protects against collective dephasing processes, while the decoupling pulses symmetrize all other sources of decoherence into a collective dephasing interaction. The recoupling pulses are used to implement encoded quantum logic gates, either during or in between the decoupling pulses.

The dynamical decoupling method requires an exponential number of pulses if the most general form of decoherence is to be suppressed, that can couple arbitrary numbers of qubits to the environment (total decoherence $\left.^{24}\right)$. This exponential scaling is avoided here by focusing on decoherence elimination inside blocks of finite size (e.g., at most four qubits) where arbitrary decoherence is allowed. However, we have implicitly assumed that there are no decoherence processes coupling different blocks. This is a reasonable assumption for most quantum 
computer implementation, provided the different blocks can be kept sufficiently far apart until they need to be brought together in order to execute inter-block logic gates. When this happens, ERD can still be efficiently applied on the temporarily larger block.

It may be questioned whether there is any advantage in using ERD compared to methods of active quantum error correcting codes (QECC). Both ERD and QECC are capable of dealing with arbitrary decoherence processes, and are fully compatible with universal quantum computation. There are two main advantages to ERD: First, we need only two physical qubits per logical qubit, compared to a redundancy of five to one, in order to handle all single-qubit errors in QECC. ${ }^{6}$ So far the most advanced experiments outside NMR, i.e., those involving trapped ions, have used up to four qubits, ${ }^{60}$ so that this encoding economy is a distinct advantage for near-term experiments. Second, our method is directly compatible with Hamiltonians describing a variety of quantum computer proposals. On the other hand it is not clear how to directly use QECC given Hamiltonians describing specific systems. These are general features of ERD: economy of encoding redundancy and use of only the most easily controllable interactions. The disadvantage of ERD compared to QECC is that there does not exist, at this point, a result analogous to the threshold theorem of fault tolerant quantum error correction. This means that we cannot yet guarantee full scalability of ERD as a stand-alone method, because we do not yet know how to compensate for imperfect pulses. However, in principle it is always possible to concatenate ERD with QECC, as done, e.g., for DFS with QECC in Refs. 66, and then the standard fault tolerance results apply. We expect that the theory of composite pulses ${ }^{67}$ will also play a key role in this further development of ERD.

We hope that the methods proposed here will inspire experimentalists to implement encoded recoupling and decoupling in the lab, thus demonstrating the possibility of fully decoherence-protected quantum computation.

\section{ACKNOWLEDGMENTS}

This material is based on research sponsored by the Defense Advanced Research Projects Agency under the QuIST program and managed by the Air Force Research Laboratory (AFOSR), under agreement F49620-01-10468. D.A.L. further gratefully acknowledges financial support from PRO, NSERC, and the Connaught Fund.

\section{REFERENCES}

1. R. Landauer, "Is quantum mechanics useful?," Proc. Roy. Soc. London Ser. A 353, p. 367, 1995.

2. W.G. Unruh, "Maintaining coherence in quantum computers," Phys. Rev. A 51, p. 992, 1995.

3. P.W. Shor, "Scheme for reducing decoherence in quantum memory," Phys. Rev. A 52, p. 2493, 1995.

4. A.M. Steane, "Error correcting codes in quantum theory," Phys. Rev. Lett. 77, p. 793, 1996.

5. A.Yu. Kitaev, "Quantum Computations: Algorithms and Error Corrections," Russian Math. Surveys 52, p. 1191, 1996.

6. R. Laflamme, C. Miquel, J.P. Paz and W.H. Zurek, "Perfect Quantum Error Correction Code," Phys. Rev. Lett. 77, p. 198, 1996.

7. J. Preskill, "Reliable quantum computers," Proc. Roy. Soc. London Ser. A 454, p. 385, 1998.

8. E. Knill, R. Laflamme and W. Zurek, "Resilient quantum computation," Science 279, p. 342, 1998.

9. A.M. Steane, "Overhead and noise threshold of fault-tolerant quantum error correction." eprint quant$\mathrm{ph} / 0207119$.

10. D. Gottesman, "Theory of fault-tolerant quantum computation," Phys. Rev. A 57, p. 127, 1997.

11. E. Knill and R. Laflamme, "Theory of quantum error-correcting codes," Phys. Rev. A 55, p. 900, 1997.

12. L.-A. Wu and D.A. Lidar, "Power of Anisotropic Exchange Interactions: Universality and Efficient Codes for Quantum Computing," Phys. Rev. A 65, p. 042318, 2002.

13. D.A. Lidar and L.-A. Wu, "Reducing Constraints on Quantum Computer Design by Encoded Selective Recoupling," Phys. Rev. Lett. 88, p. 017905, 2002.

14. L.-A. Wu and D.A. Lidar, "Qubits as Parafermions," J. Math. Phys. 43, p. 4506, 2002.

15. L.-A. Wu and D.A. Lidar, "Creating Decoherence-Free Subspaces Using Strong and Fast Pulses," Phys. Rev. Lett. 88, p. 207902, 2002.

16. M.S. Byrd and D.A. Lidar, "Comprehensive Encoding and Decoupling Solution to Problems of Decoherence and Design in Solid-State Quantum Computing," Phys. Rev. Lett. 89, p. 047901, 2002. 
17. L.-A. Wu and D.A. Lidar, "Universal Quantum Logic from Zeeman and Anisotropic Exchange Interactions," Phys. Rev. A 66, p. 062314, 2002.

18. L.-A. Wu, M.S. Byrd, D.A. Lidar, "Efficient Universal Leakage Elimination for Physical and Encoded Qubits," Phys. Rev. Lett. 89, p. 127901, 2002.

19. D.A. Lidar, L.-A. Wu, A. Blais, "Quantum Codes for Simplifying Design and Suppressing Decoherence in Superconducting Phase-Qubits," Quant. Inf. Proc. 1, p. 155, 2002.

20. L.-A. Wu, and D.A. Lidar, "Universal Quantum Computation using Exchange Interactions and Teleportation of Single-Qubit Operations," eprint quant-ph/0208118.

21. D.A. Lidar and L.-A Wu, "Encoded recoupling and decoupling: An alternative to quantum error correcting codes, applied to trapped ion quantum computation," Phys. Rev. A., in press (2003) . eprint quant$\mathrm{ph} / 0211088$.

22. P. Zanardi and M. Rasetti, "Noiseless Quantum Codes," Phys. Rev. Lett. 79, p. 3306, 1997.

23. L.-M Duan and G.-C. Guo, "Reducing decoherence in quantum-computer memory with all quantum bits coupling to the same environment," Phys. Rev. A 57, p. 737, 1998.

24. D.A. Lidar, I.L. Chuang and K.B. Whaley, "Decoherence free subspaces for quantum computation," Phys. Rev. Lett. 81, p. 2594, 1998.

25. D.A. Lidar, D. Bacon, J. Kempe, and K.B. Whaley, "Decoherence-free subspaces for multiple-qubit errors: (i) characterization," Phys. Rev. A 63, p. 022306, 2001.

26. D.A. Lidar, K.B Whaley, "Decoherence-Free Subspaces and Subsystems," 2003. eprint quant-ph/0301032.

27. L. Viola and S. Lloyd, "Dynamical suppression of decoherence in two-state quantum systems," Phys. Rev. A 58, p. 2733, 1998.

28. L.-M. Duan and G. Guo, "Suppressing environmental noise in quantum computation through pulse control," Phys. Lett. A 261, p. 139, 1999.

29. D. Vitali and P. Tombesi, "Using parity kicks for decoherence control," Phys. Rev. A 59, p. 4178, 1999.

30. D. Vitali and P. Tombesi, "Heating and decoherence suppression using decoupling techniques," Phys. Rev. A 65, p. 012305, 2002.

31. P. Zanardi, "Symmetrizing evolutions," Phys. Lett. A 258, p. 77, 1999.

32. P. Zanardi, "Computation on an error-avoiding quantum code and symmetrization," Phys. Rev. A 60, p. R729, 1999.

33. P. Zanardi, "Stabilizing quantum information," Phys. Rev. A 63, p. 012301, 2001.

34. L. Viola, E. Knill and S. Lloyd, "Dynamical decoupling of open quantum systems," Phys. Rev. Lett. 82, p. $2417,1999$.

35. L. Viola, E. Knill, and S. Lloyd, "Universal Control of Decoupled Quantum Systems," Phys. Rev. Lett. 83, p. $4888,1999$.

36. L. Viola, E. Knill, and S. Lloyd, "Dynamical Generation of Noiseless Quantum Subsystems," Phys. Rev. Lett. 85, p. 3520, 2000.

37. L. Viola, "Quantum Control via Encoded Dynamical Decoupling," Phys. Rev. A 66, p. 012307, 2002.

38. L. Viola and E. Knill, "Robust dynamical decoupling with bounded controls," Phys. Rev. Lett. 90, p. 037901, 2003.

39. G.S. Agarwal, M.O. Scully, and H. Walther, "Inhibition of Decoherence due to Decay in a Continuum," Phys. Rev. Lett. 86, p. 4271, 2001.

40. C. Search and P.R. Berman, "Suppression of Magnetic State Decoherence Using Ultrafast Optical Pulses," Phys. Rev. Lett. 85, p. 2272, 2000.

41. M.S. Byrd and D.A. Lidar, "Bang-Bang Operations from a Geometric Perspective," Quant. Inf. Proc. 1, p. 19, 2001.

42. M.S. Byrd, D.A. Lidar, "Empirical determination of dynamical decoupling operations," Phys. Rev. A 67, p. $012324,2003$.

43. M.S. Byrd and D.A. Lidar, "Combined Error Correction Techniques for Quantum Computing Architectures," J. Mod. Opt., in press (2003) . eprint quant-ph/0210072.

44. K. Shiokawa, D.A. Lidar, "Dynamical Decoupling Using Slow Pulses: Efficient Suppression of 1/f Noise," 2002. eprint quant-ph/0211081. 
45. C. Uchiyama and M. Aihara, "Multipulse control of decoherence," Phys. Rev. A 66, p. 032313, 2002.

46. D. Bacon, J. Kempe, D.A. Lidar and K.B. Whaley, "Universal Fault-Tolerant Computation on DecoherenceFree Subspaces," Phys. Rev. Lett. 85, p. 1758, 2000.

47. J. Kempe, D. Bacon, D.A. Lidar, and K.B. Whaley, "Theory of Decoherence-Free, Fault-Tolerant, Universal Quantum Computation," Phys. Rev. A 63, p. 042307, 2001.

48. D.A. Lidar, Z. Bihary, and K.B. Whaley, "From Completely Positive Maps to the Quantum Markovian Semigroup Master Equation," Chem. Phys. 268, p. 35, 2001.

49. P.G. Kwiat, A.J. Berglund, J.B. Altepeter, and A.G. White, "Experimental Verification of Decoherence-Free Subspaces," Science 290, p. 498, 2000.

50. D. Kielpinski, V. Meyer, M.A. Rowe, C.A. Sackett, W.M. Itano, C. Monroe, and D.J. Wineland, "A Decoherence-Free Quantum Memory Using Trapped Ions," Science 291, p. 1013, 2001.

51. J. Levy, "Universal quantum computation with spin-1/2 pairs and Heisenberg exchange," Phys. Rev. Lett. 89, p. 147902, 2002.

52. S.C. Benjamin, "Simple Pulses for Universal Quantum Computation with a Heisenberg ABAB Chain," Phys. Rev. A 64, p. 054303, 2001.

53. D.P. DiVincenzo, D. Bacon, J. Kempe, G. Burkard, and K.B. Whaley, "Universal Quantum Computation with the Exchange Interaction," Nature 408, p. 339, 2000.

54. J. Kempe, D. Bacon, D.P. DiVincenzo and K.B. Whaley, "Encoded Universality from a Single Physical Interaction," Quant. Inf. Comp. 1, p. 33, 2001.

55. J. Kempe and K.B. Whaley, "Exact gate-sequences for universal quantum computation using the XYinteraction alone," Phys. Rev. A 65, p. 052330, 2001.

56. J. Vala and K.B. Whaley, "Encoded Universality for Generalized Anisotropic Exchange Hamiltonians," Phys. Rev. A 66, p. 022304, 2002.

57. D. Bacon, J. Kempe, D.P. DiVincenzo, D.A. Lidar, and K.B. Whaley, "Encoded universality in physical implementations of a quantum computer," in Proceedings of the 1st International Conference on Experimental Implementations of Quantum Computation, Sydney, Australia, R. Clark, ed., p. 257, Rinton, (Princeton, NJ), 2001.

58. D. J. Wineland, C. Monroe, W. M. Itano, D. Leibfried, B. E. King, and D. M. Meekhof, "Experimental Issues in Coherent Quantum-State Manipulation of Trapped Atomic Ions," J. of Res. of the National Inst. of Standards and Technology 103, p. 259, 1998. http://nvl.nist.gov/pub/nistpubs/jres/103/3/cnt103-3.htm.

59. A. Sørensen and K. Mølmer, "Entanglement and quantum computation with ions in thermal motion," Phys. Rev. A 62, p. 022311, 2000.

60. C. A. Sackett, D. Kielpinski, B. E. King, C. Langer, V. Meyer, C. J. Myatt, M. Rowe, Q. A. Turchette, W. M. Itano, D. J. Wineland, and C. Monroe, "Experimental entanglement of four particles," Nature 404, p. $256,2000$.

61. D. Kielpinski, C. Monroe and D.J. Wineland, "Architecture for a large-scale ion-trap quantum computer," Nature 417, p. 709, 2002.

62. M.A. Nielsen and I.L. Chuang, Quantum Computation and Quantum Information, Cambridge University Press, Cambridge, UK, 2000.

63. R.R. Ernst, G. Bodenhausen and A. Wokaun, Principles of Nuclear Magnetic Resonance in One and Two Dimensions, no. 14 in International Series of Monographs on Chemistry, Oxford University Press, Oxford, 1997.

64. J.P. Palao and R. Kosloff, "Quantum Computing by an Optimal Control Algorithm for Unitary Transformations," Phys. Rev. Lett. 89, p. 188301, 2002.

65. L. Tian and S. Lloyd, "Resonant cancellation of off-resonant effects in a multilevel qubit," Phys. Rev. A 62, p. 050301, 2000.

66. K. Khodjasteh and D.A. Lidar, "Universal Fault-Tolerant Quantum Computation in the Presence of Spontaneous Emission and Collective Dephasing," Phys. Rev. Lett. 89, p. 197904, 2002.

67. H.K. Cummins, G. Llewellyn, J.A. Jones, "Tackling Systematic Errors in Quantum Logic Gates with Composite Rotations," eprint quant-ph/0208092. 